ANÁLISIS COMPARATIVO DE LOS ESTÁNDARES DE CALIDAD DE UNA EMPRESA MEXICANA CON LAS PRINCIPALES TEORÍAS DE LA CALIDAD ENFOCADAS EN EL SERVICIO AL CLIENTE.

\title{
ANÁLISIS COMPARATIVO DE LOS ESTÁNDARES DE CALIDAD DE UNA EMPRESA NACIONAL CON LAS PRINCIPALES TEORÍAS DE LA CALIDAD ENFOCADAS EN EL SERVICIO AL CLIENTE
}

\section{COMPARATIVE ANALYSIS OF QUALITY STANDARDS OF A NATIONAL COMPANY WITH MAIN QUALITY THEORIES FOCUSED ON CUSTOMER SERVICE}

Alpha Díaz García* Cecilia García Muñoz Aparicio**

*Pasante de la Licenciatura en Administración en la División Académica de Ciencias Económico Administrativas de la Universidad Juárez Autónoma de Tabasco. ORCID: https://orcid.org/0000-00024354-8768.

** Doctora en Ciencias Económico Administrativas. División Académica de Ciencias Económico Administrativas. Universidad Juárez Autónoma de Tabasco. Email: flamingos1999@hotmail.com. ORCID: https://orcid.org/0000-0001-5316-8630.

Dirección para correspondencia: alphadiaz8@gmail.com 
ANÁLISIS COMPARATIVO DE LOS ESTÁNDARES DE CALIDAD DE UNA EMPRESA MEXICANA CON LAS PRINCIPALES TEORÍAS DE LA CALIDAD ENFOCADAS EN EL SERVICIO AL CLIENTE.

\title{
RESUMEN
}

OBJETIVO: Analizar si los estándares de calidad de una empresa nacional se basan en las teorías clásicas de la calidad además de compararlas con cada uno de los estándares desarrollados por la empresa. Todo esto con enfoque específico en el servicio al cliente.

MATERIAL Y MÉTODO: El estudio se considera exploratorio, comparativo con enfoque cualitativo aplicado hacia una empresa mexicana del sector privado, creando una comparación entre las teorías de la calidad clásicas y la información obtenida sobre los estándares de servicio al cliente brindado por el talento humano.

RESULTADOS: Se hallaron similitudes positivas y negativas entre ambos objetos de estudio, además de encontrar diferencias entre las teorías formuladas por los gurús de la calidad.

CONCLUSIÓN: Según los análisis comparativo y crítico realizados, la empresa mexicana presentó un proceso de ventas ad hoc a las filosofías de los pioneros de la calidad; sin embargo, no se confirma que el fundamento de sus estándares sean las teorías presentadas, además se hace énfasis en la importancia de investigar la calidad en el servicio al cliente a profundidad y se menciona la necesidad de mejorar este aspecto tan importante para los compradores en el estado y el país.

PALABRAS CLAVE: Calidad. Estándares. Teorías de la calidad. Servicio al cliente.

\begin{abstract}
OBJECTIVE: To analyze if quality standards of a national company are based on classical quality theories besides comparing them with each of the company standards developed by the enterprise. All this with a specific approach in customer service.
\end{abstract}

MATERIAL AND METHOD: The study is exploratory, comparative with a qualitative approach in a Mexican enterprise from the private sector, creating a comparison between classical quality theories and the information obtained about the service standards given to clients by the human talent. 
ANÁLISIS COMPARATIVO DE LOS ESTÁNDARES DE CALIDAD DE UNA EMPRESA MEXICANA CON LAS PRINCIPALES TEORÍAS DE LA CALIDAD ENFOCADAS EN EL SERVICIO AL CLIENTE.

RESULTS: Positive and negative similarities were found in both objects of study, besides the differences between the formulated theories from gurus in quality.

CONCLUSIONS: According to comparative and critical analysis performed, the Mexican company presented a sales process ad hoc to philosophies from quality pioneers, however, it is not confirmed the fundamentals on their standards are the previously presented theories, besides an emphasis on the importance of doing in-depth quality research in customer services and it is mentioned the necessity of improving this important aspect for buyers in the state and in the country.

KEY WORDS: Quality, standards, quality theories, customer service.

\section{INTRODUCCIÓN}

La calidad es una característica subjetiva que se puede lograr en diversos sectores de una empresa. Se fundamenta directamente en la satisfacción del cliente, como una herramienta que incentiva la venta del producto para que adquiera un mayor valor agregado y que el servicio brindado tenga mayor impacto en el cliente, según sea el caso. "Muchas organizaciones grandes y pequeñas han llegado a la conclusión de que la efectividad de la dirección de la calidad puede reforzar su competitividad y proporcionar ventajas competitivas en el mercado" (Anderson, Rungtusanatham y Schroeder, 1994; Sitkin, Sutcliffe y Schroeder, 1994).

Aunque México aún no ha demostrado ser un rival para países como Japón, Alemania o Estados Unidos en cuestiones de calidad, la competitividad ha hecho que las empresas nacionales evolucionen y se preocupen más por ofrecer una mayor calidad tanto en la producción como en el servicio al cliente interno (los empleados) y externo (el mercado). La necesidad de implantar un sistema de calidad funcional dentro de una organización, es tan primordial que no solo se aplica a los procesos productivos o de gestión, sino también, al recurso más importante dentro de la empresa: el personal (talento humano).

Este es el caso de la empresa mexicana estudiada, ubicada en el giro comercial, la cual ha diseñado nueve estándares que sus empleados deben realizar para garantizar la venta de sus productos y al mismo tiempo brindar un servicio de calidad que manifieste una diferencia en la 
ANÁLISIS COMPARATIVO DE LOS ESTÁNDARES DE CALIDAD DE UNA EMPRESA MEXICANA CON LAS PRINCIPALES TEORÍAS DE LA CALIDAD ENFOCADAS EN EL SERVICIO AL CLIENTE.

competencia nacional. Los estándares conocidos como los Nueve Estándares No Negociables (9ENN), abarcan en el servicio al cliente desde el saludo de bienvenida, hasta que el cliente se retira de la tienda habiendo, o no, adquirido un producto.

Por lo antes mencionado, se realizará un estudio comparativo para identificar si los 9ENN se apegan a las teorías de la calidad que los pioneros han implementado desde hace décadas y que, en la actualidad, muchas empresas las implementan en sus técnicas de venta diarias. Cabe mencionar, que el enfoque de cada una de las teorías es completamente dirigido al servicio al cliente. Por ello se hará uso de teorías cualitativas que sirvan como comparativa con los estándares a analizar.

\section{DESARROLLO}

\section{La calidad y el cliente}

La calidad tiene diferentes significados según sea el área desde la cual se califique la misma. Según Juran (1990), la palabra calidad tiene múltiples significados, los más representativo son:

1. Es la característica de un producto con base en las necesidades del cliente, brindando satisfacción al mismo.

2. Es la libertad después de las deficiencias.

Por su parte Ishikawa (1986), uno de los íconos más reconocidos de la calidad, afirma: "de manera sintética, calidad significa calidad del producto. Más específicamente, calidad es calidad del trabajo, calidad del servicio, calidad de la información, calidad de proceso, calidad de la gente, calidad del sistema, calidad de la compañía, calidad de objetivos".

La calidad abarca cada uno de los rincones de una empresa, ya sea de manufactura o comercial, lo cual se resume en determinar las necesidades de los clientes y su satisfacción, tal como lo indica Deming (1989):

Calidad es traducir las necesidades futuras de los usuarios en características medibles; solo así un producto puede ser diseñado y fabricado para dar satisfacción a un precio que el cliente pagará; la calidad puede estar definida solamente en términos del agente.

Por último, se presenta la definición de Crosby (1988), para quien "calidad es conformidad con los requerimientos, los cuales tienen que estar establecidos de forma clara para que no existan 
ANÁLISIS COMPARATIVO DE LOS ESTÁNDARES DE CALIDAD DE UNA EMPRESA MEXICANA CON LAS PRINCIPALES TEORÍAS DE LA CALIDAD ENFOCADAS EN EL SERVICIO AL CLIENTE.

malentendidos; las mediciones deben ser tomadas de manera continua para determinar dichos requerimientos; la no conformidad detectada es una ausencia de calidad".

La calidad es el cumplimiento de las expectativas del cliente de forma eficiente y eficaz. Para entender mejor este concepto, se requiere establecer que la eficacia es el cumplimiento de los objetivos propuestos, mientras que la eficiencia se apega a la optimización de los recursos, es decir lograr los resultados deseados con el mínimo de tiempo y recursos. Para una empresa es primordial conocer estos dos conceptos si uno de sus objetivos es alcanzar la calidad.

Por otra parte, Schnarch (2011, p. 13) considera que un cliente extremadamente insatisfecho es un enemigo en el futuro, uno medianamente insatisfecho es un cliente que puede cambiar su opinión mientras que un cliente satisfecho puede retenerse o perderse; solamente un cliente extremadamente satisfecho es un promotor para el negocio.

Dicho de otra manera, el cliente puede ser un fiel aliado de la empresa, siempre y cuando se le mantenga en un adecuado grado de satisfacción de expectativas y necesidades. La importancia de cuidar el trato hacia los clientes se debe a que en la actualidad es más sencillo compartir una opinión personal con otras personas acerca del servicio de algún establecimiento u organización, sobre todo en las redes sociales cuya información es transmitida de manera casi inmediata,

En la figura 1, se muestran las diversas causas por las cuales se puede perder a un cliente; sin embargo, el mayor porcentaje está determinado por factores relacionados con la empresa y la atención que éste recibe.

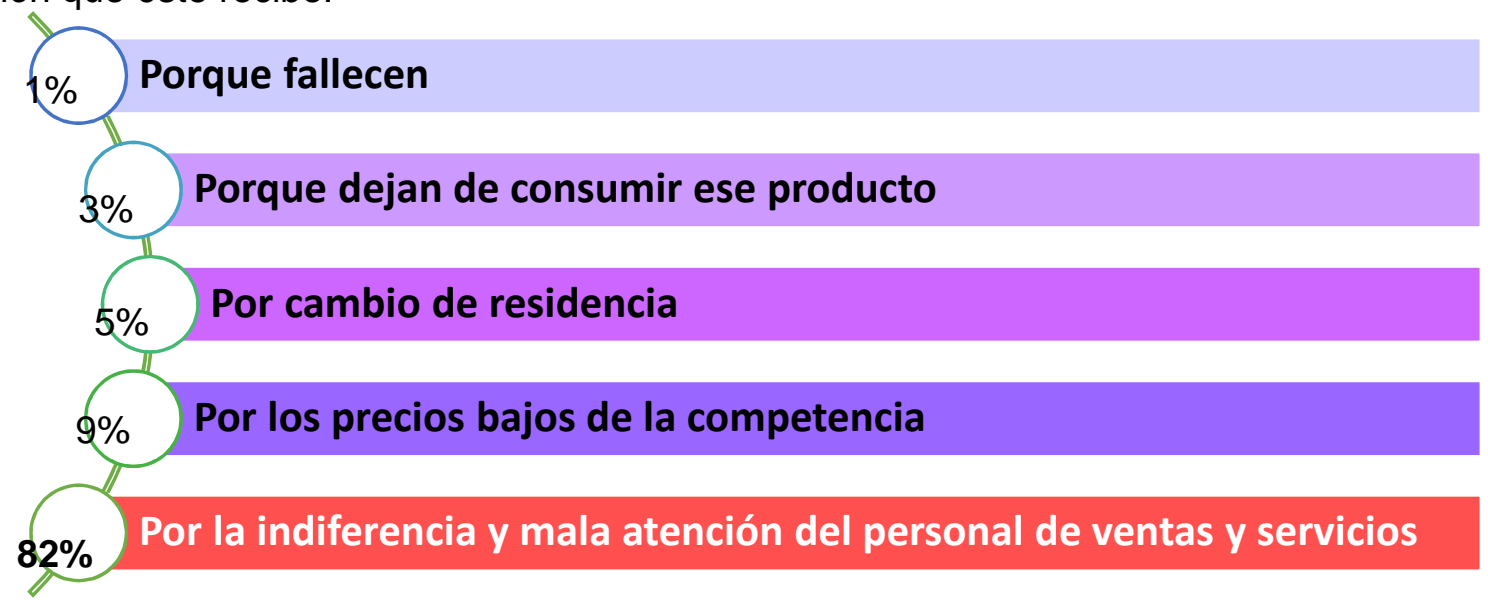

\section{Figura 1. ¿Por qué se pierden los clientes?}

Fuente: Elaboración con base en Hatch (2019). 
ANÁLISIS COMPARATIVO DE LOS ESTÁNDARES DE CALIDAD DE UNA EMPRESA MEXICANA CON LAS PRINCIPALES TEORÍAS DE LA CALIDAD ENFOCADAS EN EL SERVICIO AL CLIENTE.

En esta figura se observan los resultados de un estudio de redes sociales elaborado por Hatch (2019), el estudio arrojó un $82 \%$ de clientes perdidos por la mala atención, observando como el porcentaje se incrementa debido a las redes sociales. Los clientes reamente se pierden debido a la indiferencia con la que son tratados y la mala atención por parte del personal.

\section{Teorías y modelos de la calidad}

Las teorías y modelos clásicos de calidad, servirán como pauta para desarrollar la investigación, puesto que se tomará la información necesaria para realizar los análisis comparativos, resultados y conclusiones. En la tabla 1 se observan las aportaciones y conceptos de los autores clásicos de calidad relacionados con el servicio al cliente.

Tabla 1

Teorías y modelos clásicos de calidad

\begin{tabular}{|c|c|c|}
\hline Investigador & Aportaciones & $\begin{array}{c}\text { Conceptos básicos con relación al servicio al } \\
\text { cliente }\end{array}$ \\
\hline $\begin{array}{l}\text { Edward Deming } \\
(1900-1993), \text { físico } \\
\text { y estadístico } \\
\text { estadounidense. }\end{array}$ & $\begin{array}{l}\text { - } 14 \text { Principios de } \\
\text { Deming } \\
\text { Ciclo PHVA } \\
\text { (Planear, Hacer, } \\
\text { Verificar Y Actuar) }\end{array}$ & $\begin{array}{l}\text { - Aplicar la calidad en todos los aspectos de } \\
\text { la empresa, motivar al talento humano y } \\
\text { mejorar el servicio al cliente. } \\
\text { - Fortalecer el proceso de mejora continua. }\end{array}$ \\
\hline $\begin{array}{l}\text { Shigeo Shingo } \\
(1909-1990), \\
\text { ingeniero industrial } \\
\text { japonés. }\end{array}$ & - Metodología 9's & $\begin{array}{l}\text { - Implementar y cuidar del orden y limpieza } \\
\text { de todas las áreas laborales. }\end{array}$ \\
\hline $\begin{array}{l}\text { Philip Crosby } \\
(1926-2001) \\
\text { empresario } \\
\text { estadounidense. }\end{array}$ & $\begin{array}{l}\text { Cuatro principios } \\
\text { absolutos } \\
14 \text { pasos para la } \\
\text { calidad. }\end{array}$ & $\begin{array}{l}\text { - Establecer un estándar de cero defectos y } \\
\text { de no conformidad. } \\
\text { - Se consideran todos los aspectos, tanto } \\
\text { internos como externos para lograr la } \\
\text { calidad con cero defectos. }\end{array}$ \\
\hline $\begin{array}{l}\text { Joseph M. Juran } \\
\text { (1904-2008), } \\
\text { ingeniero y } \\
\text { abogado rumano. }\end{array}$ & - La trilogía de Juran. & $\begin{array}{l}\text { - Adecuar la calidad al uso, es decir adaptar } \\
\text { el servicio de acuerdo al tipo de cliente. }\end{array}$ \\
\hline $\begin{array}{l}\text { Kaoru Ishikawa } \\
\text { (1915-1989), } \\
\text { ingeniero, } \\
\text { catedrático y } \\
\text { consultor japonés. }\end{array}$ & $\begin{array}{l}\text { - Principios básicos de } \\
\text { la calidad. } \\
\text { Las } 7 \text { herramientas } \\
\text { básicas de calidad } \\
\text { (Diagrama Causa- } \\
\text { Efecto). }\end{array}$ & $\begin{array}{l}\text { - Aplicar la calidad en todo momento, incluso } \\
\text { después de la compra, cuidar cada aspecto } \\
\text { de la atención que se brinda al cliente } \\
\text { asegura la fidelidad y la satisfacción del } \\
\text { mismo } \\
\text { - Secuenciar el servicio al cliente desde que } \\
\text { entra a la tienda hasta que sale de ella. }\end{array}$ \\
\hline
\end{tabular}

Fuente: Elaboración con base en Alvarado y Rivas, (2006), Chacón y Rugel, (2018), Ojeda y López, (2000), Ruiz, Fernández y Tamaro (2004), y Tovar, (2017). 
ANÁLISIS COMPARATIVO DE LOS ESTÁNDARES DE CALIDAD DE UNA EMPRESA MEXICANA CON LAS PRINCIPALES TEORÍAS DE LA CALIDAD ENFOCADAS EN EL SERVICIO AL CLIENTE.

En la tabla 1, se muestra de forma resumida la relación que existe entre las teorías clásicas de la calidad y la calidad en el servicio al cliente. Cada teoría y filosofía de los autores clásicos, se adapta al proceso de atención que un trabajador brinda a los compradores.

\section{La empresa}

Empresa mexicana Prada, cuyo fundador es español y sus productos son provenientes de Europa, se encarga de comercializar accesorios de piel vacuna y caprina, como son: bolsas, carteras, zapatos, monederos, cinturones; cuya misión es "exaltar la imagen con experiencias memorables" (Barajas, 2013). Se ubicada dentro de las marcas Premium en México.

Según la información de Esencia Prada (s.f.), en el año de 1951 llegó a México el fundador de la compañía, con el objetivo de unir las visiones españolas y mexicanas en relación a la elegancia y el buen gusto por el calzado para varias generaciones. En 1956, funda su primera marca de calzado y accesorios la cual, con el paso del tiempo, llegó a ser una sociedad que agrupaba más de 40 tiendas y 3 fábricas, mismas que vendió en el año 2003. En 1997, se funda una nueva cadena de accesorios de piel, mejorando su productividad y sus diseños, transformando la exclusividad de la marca. En el año 2018, la empresa se posicionó como la mejor marca Premium de su segmento, logrando aperturar 30 boutiques y 5 outlets dentro de la República Mexicana.

\section{Características de la empresa}

Todas las boutiques se encuentran estandarizadas bajo un solo diseño arquitectónico, con la finalidad que los clientes puedan viajar de una sucursal a otra sin sentir diferencia alguna. La estructura cuenta con el piso de ventas, la pre-bodega, la zona de comida, la bodega y al final el sanitario exclusivo del personal. Cada boutique cuenta con su licencia de funcionamiento correspondiente y es visitada cada año por Protección Civil, para verificar que cumpla con las normas de seguridad e higiene.

Las funciones varían dependiendo el puesto. Para la investigación el enfoque se realizó hacia los asesores de venta, quienes llevan a la práctica los 9 Estándares No Negociables (9ENN). Las actividades de un asesor de ventas son la atención al cliente, la limpieza y el acomodo de bodega. 
ANÁLISIS COMPARATIVO DE LOS ESTÁNDARES DE CALIDAD DE UNA EMPRESA MEXICANA CON LAS PRINCIPALES TEORÍAS DE LA CALIDAD ENFOCADAS EN EL SERVICIO AL CLIENTE.

En atención al cliente, se espera que el asesor brinde una experiencia memorable, además de agregar adicionales, como ofrecer diferentes servicios y el programa de fidelidad de la empresa. La limpieza del área de responsabilidad va por cuenta de cada asesor, según la que haya sido asignada por el gerente de la boutique. Por último, el acomodo de bodega debe realizarse de manera periódica y se deben seguir las políticas de acomodo establecidas por la empresa.

Una de las responsabilidades del asesor de ventas es conocer el producto, para ello, se les proporciona un manual en el cual se establecen las partes de cada producto, los materiales con los que son fabricados, los métodos de producción y las líneas especiales con las que se cuenta.

De esta manera, cada empleado debe cumplir con las normas internas generales de la boutique, que van desde la puntualidad en la hora de entrada y salida, la hora de comida, el comportamiento, el uso correcto del uniforme, la restricción de los teléfonos, conocer las políticas sobre el esquema de pago y tomar las capacitaciones por medio de la plataforma personalizada de la empresa.

Para la investigación, se tomaron en cuenta las características de los 9ENN, enlistadas y detalladas en la tabla 2, obtenidas en la documentación que la empresa proporciona a sus empleados.

Tabla 2

Los Nueve Estándares No Negociables (9ENN)

\begin{tabular}{lll}
\hline \multicolumn{2}{c}{ Estándar No Negociable } & \multicolumn{1}{c}{ Características } \\
\hline $\begin{array}{l}\text { 1. Presentación } \\
\text { comportamiento }\end{array}$ & - & Porta uniforme, gafete, peinado, maquillaje y accesorios de \\
& acuerdo al código de vestimenta \\
& - No celular en el piso de ventas. \\
& - No comida, ni bebida en el piso de ventas \\
& - No vistas personales durante la jornada de trabajo \\
& - No comportamiento lúdico y afectivo \\
& - & Saludar sonriendo haciendo contacto visual evidente, dentro de \\
& los primeros 5 segundos a partir de que el cliente ingresa a \\
& sucursal. \\
2. Saludar al cliente & Si todo el personal de sucursal se encuentra con clientes, la \\
& persona que le da la bienvenida deberá hacer una señal física \\
& que indique: que en seguida será atendido (ejemplo señalarse \\
& el reloj o hacer el gesto de espera de tiempo con los dedos).
\end{tabular}

(continúa) 
ANÁLISIS COMPARATIVO DE LOS ESTÁNDARES DE CALIDAD DE UNA EMPRESA MEXICANA CON LAS PRINCIPALES TEORÍAS DE LA CALIDAD ENFOCADAS EN EL SERVICIO AL CLIENTE.

\section{Tabla 2}

Los Nueve Estándares No Negociables (9ENN)

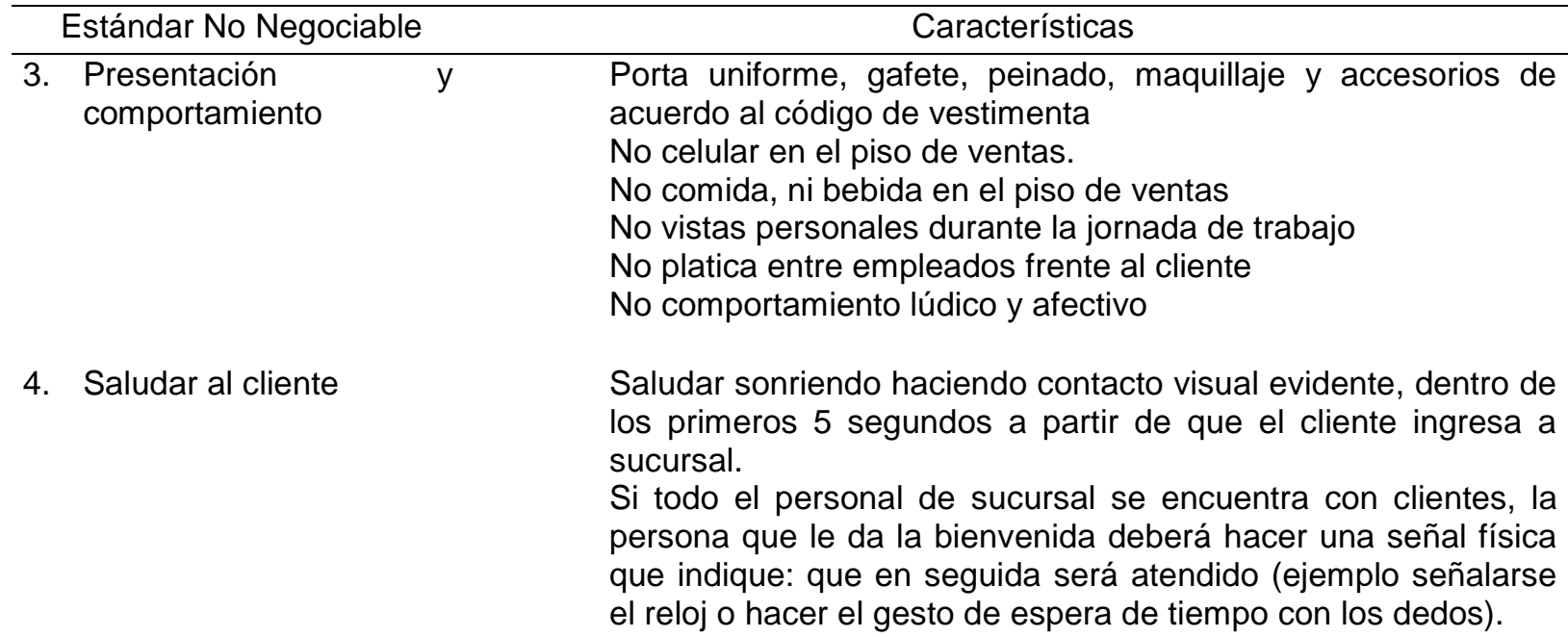

5. Preguntas investigación

de

roductos al

7. Adicionar

8. Darle valor al producto

9. Programa de fidelidad

10. Cobro

11. Despedir al cliente
- Realizar como mínimo 3 preguntas de investigación, con la finalidad de detectar lo que el cliente está buscando y poder mostrárselo

- Es importante formular preguntas abiertas para averiguar más acerca de los gustos y necesidades de un cliente

- El personal deberá mostrar 3 artículos del mismo género al cliente

- Mostrar artículos diferentes al que mostró en un principio

- Hacer una presentación frente al cliente de cada producto que muestre, mencionando sus características, sus ventajas y sus beneficios

- Explicarle al cliente sobre el programa de fidelidad de la empresa e invitarlo a formar parte de él

- Revisar el producto (modelo, color y talla)

- Entregar al cliente sobre con el ticket y tarjeta de presentación del vendedor

- Reafirmar el programa de fidelidad

- Acompañar al cliente a la puerta, cargándole el producto.

- Despedir, agradecer e invitar a que regrese.

Fuente: Elaboración con base en los datos del documento Estándares de venta de Barajas (2011).

Estos estándares, se aplican al realizar la labor de venta con cada cliente que visita una boutique y de acuerdo con Barajas (2011) su función es "estandarizar el proceso de ventas y 
ANÁLISIS COMPARATIVO DE LOS ESTÁNDARES DE CALIDAD DE UNA EMPRESA MEXICANA CON LAS PRINCIPALES TEORÍAS DE LA CALIDAD ENFOCADAS EN EL SERVICIO AL CLIENTE.

brindar un servicio de calidad al cliente e incrementar las ventas". Aunque son los asesores de venta quienes aplican los $9 \mathrm{ENN}$, todos los empleados los deben conocer ya que son obligatorios.

\section{MATERIAL Y MÉTODO}

Para Aguilera, (2011), la metodología es importante debido a que se estudian elementos relacionados con el origen, fundamentación, articulación ética, razonabilidad; la capacidad explicativa, la utilidad aplicada y los procedimientos de control que se utilizan en algún trabajo.

Esta investigación es exploratoria debido a que "examina un tema o problema de investigación poco estudiado, del cual se tienen muchas dudas o no se ha abordado antes" (Hernández, Fernández y Baptista, 2014, p. 91), ya que la calidad es un tema que se enfoca en su mayoría hacia la producción y a sus procesos. De igual forma, se considera un estudio comparativo pues Díaz y De León, (2014) indican que "el objetivo fundamental del método comparativo consiste en la generalización empírica y la verificación de hipótesis" lo cual es la finalidad de esta investigación.

Se puede determinar que la investigación posee un enfoque cualitativo debido a que "la investigación cualitativa trata de identificar la naturaleza profunda de las realidades, su estructura dinámica, aquella que da razón plena de su comportamiento y manifestaciones" (Martínez, 2019) y el objetivo principal de la investigación es comparar la esencia de ambos objetos de estudio, además que los datos que se tomaron en cuenta son basados en comportamientos y experiencias de vivencias personales de los estándares aplicados por los asesores de la empresa.

Primero, se describieron las teorías, posteriormente se analizaron los estándares de servicio durante el proceso de venta y al final se realizó un cuadro comparativo por cada uno de los 9ENN, comparándolos con las teorías. La comparación se realizó de forma cualitativa, analítica y descriptiva.

\section{RESULTADOS}

Los resultados son una serie de cuadros comparativos, los cuales están divididos en dos columnas: en la primera se encuentra la o las teorías con las que se encontró afinidad, sea positiva o negativamente y por último una breve conclusión del análisis comparativo; dicha DÍAZ-GARCÍA A., GARCÍA-MUÑOZ APARICIO C. 
ANÁLISIS COMPARATIVO DE LOS ESTÁNDARES DE CALIDAD DE UNA EMPRESA MEXICANA CON LAS PRINCIPALES TEORÍAS DE LA CALIDAD ENFOCADAS EN EL SERVICIO AL CLIENTE.

relación se describe por cada teoría, es decir, cada número señalado en la fila "Teoría(s) para comparar" corresponde con el número marcado en la fila "Análisis comparativo". Por ejemplo, la relación de la teoría número 1 con el estándar señalado, es explicada en el análisis número 1 y así sucesivamente.

\section{Tabla 3}

Estándar 1: Presentación y comportamiento

\begin{tabular}{|c|c|}
\hline Teoría(s) para comparar & Análisis comparativo \\
\hline 1. 14 Principios de Deming & $\begin{array}{l}\text { 1. Uno de los principios de Edward Deming es "dejar de depender de la } \\
\text { inspección en masa para lograr la calidad" y en este caso la empresa } \\
\text { mantiene una inspección constante de las políticas del primer estándar, lo } \\
\text { cual recae en lo que Deming califica como innecesario }\end{array}$ \\
\hline 2. Ciclo PHVA (Planear) & $\begin{array}{l}\text { 2. La relación que existe es en la planificación de la recepción del cliente, según } \\
\text { el estándar se debe estar preparado para su llegada en todo momento }\end{array}$ \\
\hline 3. Metodología 9's & $\begin{array}{l}\text { 3. Como se establece en el estándar, todo debe estar en perfecto orden al } \\
\text { entrar a la tienda, así como la higiene y cuidado del personal mismo }\end{array}$ \\
\hline
\end{tabular}

Fuente: Elaboración propia con base en Castillo, (2016) y Miranda, Chamorro y Rubio, (2007).

La tabla 3, explica la relación con tres de las teorías en donde se observan comportamientos innecesarios para la productividad y a la vez, se aprecia la planificación que existe hacia el proceso de ventas aún sin contemplar el contacto con los clientes en el primer estándar.

\section{Tabla 4}

Estándar 2: Saludar al cliente

\begin{tabular}{|c|c|}
\hline Teoría(s) para comparar & Análisis comparativo \\
\hline 1. Ciclo PHVA (Planear) & $\begin{array}{l}\text { 1. Este estándar se ubica de la misma manera en la planeación, en conocer las } \\
\text { acciones para con el cliente desde los primeros segundos de su entrada a la } \\
\text { boutique }\end{array}$ \\
\hline $\begin{array}{l}\text { 2. } 14 \text { pasos para la calidad } \\
\text { de Crosby }\end{array}$ & $\begin{array}{l}\text { 2. En algunos de sus pasos para la calidad Crosby hace mención a las medidas } \\
\text { de la calidad y la planificación cero defectos, esto se toma en cuenta cuando } \\
\text { un asesor recibe al cliente pues, aunque se encuentre ocupado, debe actuar } \\
\text { de manera cordial ofreciendo el servicio lo más pronto posible }\end{array}$ \\
\hline 3. Trilogía de Juran & $\begin{array}{l}\text { 3. La trilogía de Juran en su primer paso menciona que se debe planear la } \\
\text { calidad, se deben reconocer a los clientes desde su entrada a la boutique } \\
\text { para poder ofrecerle la atención ideal }\end{array}$ \\
\hline $\begin{array}{l}\text { 4. Principios básicos de la } \\
\text { calidad de Ishikawa }\end{array}$ & $\begin{array}{l}\text { 4. Como menciona Ishikawa en su primer principio básico de la calidad "la } \\
\text { calidad empieza con educación" por ello es fundamental que cada asesor } \\
\text { cuente con }\end{array}$ \\
\hline
\end{tabular}

Fuente: Elaboración propia con base en Chacón y Rugel, (2018) y Miranda, Chamorro y Rubio, (2007). 
ANÁLISIS COMPARATIVO DE LOS ESTÁNDARES DE CALIDAD DE UNA EMPRESA MEXICANA CON LAS PRINCIPALES TEORÍAS DE LA CALIDAD ENFOCADAS EN EL SERVICIO AL CLIENTE.

En la tabla 4, se aprecia que el segundo estándar se identifica nuevamente como un inicio o planeación para las teorías, este es el verdadero primer contacto que el asesor tiene con el cliente dentro de la boutique.

\section{Tabla 5}

Estándar 3: Preguntas de investigación

\begin{tabular}{|c|c|c|c|}
\hline \multirow{2}{*}{\multicolumn{2}{|c|}{$\begin{array}{l}\text { Teoría(s) para comparar } \\
\text { Ciclo PHVA (Hacer) }\end{array}$}} & \multicolumn{2}{|r|}{ Análisis comparativo } \\
\hline & & 1. & $\begin{array}{l}\text { Para Deming el "hacer" es identificar el problema, con las preguntas de } \\
\text { investigación correctas el asesor puede conocer lo que el cliente quiere y así } \\
\text { ofrecerle el producto adecuado para su satisfacción. }\end{array}$ \\
\hline 2. & $\begin{array}{l}4 \text { principios absolutos } \\
\text { de Crosby }\end{array}$ & 2. & $\begin{array}{l}\text { En su tercer principio absoluto Crosby establece que se debe acordar con los } \\
\text { empleados el resultado que la dirección desea y en este caso la empresa } \\
\text { establece un estándar de mínimo tres preguntas de investigación para } \\
\text { descubrir lo que el cliente desea, esto debe cumplirse, aunque la compra no } \\
\text { se lleve a cabo. }\end{array}$ \\
\hline 3. & $\begin{array}{l}14 \text { pasos de la calidad } \\
\text { de Crosby }\end{array}$ & 3. & $\begin{array}{l}\text { Al ser este estándar el primero donde los clientes pueden hacer objeciones } \\
\text { los asesores deben contar con el conocimiento necesario para responder a } \\
\text { cualquiera de ellas, tal como Crosby indica "definir un programa de actuación } \\
\text { para la prevención de errores" (paso 7), además que anterior al proceso de } \\
\text { venta ya existió una capacitación completa (paso 8) para erradicar los } \\
\text { impedimentos que reduzcan la efectividad de dicha capacitación (paso 11). }\end{array}$ \\
\hline 4. & Trilogía de Juran & 4. & $\begin{array}{l}\text { Este estándar en particular abarca la planificación de la calidad y el control de } \\
\text { la calidad basándonos en lo que menciona Juran, ya que desde las preguntas } \\
\text { que se realicen se identifica más a fondo el tipo de cliente con el que se trata } \\
\text { y se puede desarrollar un servicio más acorde a él además de evaluar su } \\
\text { comportamiento para poder cumplir el objetivo de la venta. }\end{array}$ \\
\hline 5. & $\begin{array}{l}\text { Las } 7 \text { herramientas de } \\
\text { calidad (Diagrama de } \\
\text { flujo }\end{array}$ & 5. & $\begin{array}{l}\text { Este estándar en específico es uno de los cruciales para el proceso de venta } \\
\text { de un asesor, ya que la información obtenida y sus respuestas hacia el cliente } \\
\text { definirán si la compra se llevará a cabo o no. }\end{array}$ \\
\hline
\end{tabular}

Fuente: Elaboración propia con base en Marmolejo (2019) y Miranda, Chamorro y Rubio, (2007).

Se identifica al tercer estándar como el más importante de los 9ENN, debido a que este es el que logrará definir el rumbo del proceso de ventas y el que hará la diferencia en el servicio que perciba el cliente. 
ANÁLISIS COMPARATIVO DE LOS ESTÁNDARES DE CALIDAD DE UNA EMPRESA MEXICANA CON LAS PRINCIPALES TEORÍAS DE LA CALIDAD ENFOCADAS EN EL SERVICIO AL CLIENTE.

\section{Tabla 6}

Estándar 4: Mostrar 3 productos al cliente

\begin{tabular}{|c|c|}
\hline Teoría(s) para comparar & Análisis comparativo \\
\hline 1. Ciclo PHVA (Verificar) & $\begin{array}{l}\text { 1. En el verificar del ciclo PHVA se manifiesta que se debe proceder con } \\
\text { respecto a las políticas y a los objetivos marcados, en este estándar se } \\
\text { muestran los productos de acuerdo a los gustos y especificaciones del } \\
\text { cliente, además de ofrecer lo más nuevo y novedoso que se encuentre en la } \\
\text { empresa }\end{array}$ \\
\hline 2. Metodología 9's & $\begin{array}{l}\text { 2. La limpieza y el orden de las bodegas harán más rápida la búsqueda de un } \\
\text { producto para mostrar al cliente, sin mencionar que el que se vaya a mostrar } \\
\text { debe estar en perfectas condiciones }\end{array}$ \\
\hline 3. Trilogía de Juran & $\begin{array}{l}\text { 3. En su segundo punto de la trilogía, Juran indica que "se debe actuar sobre las } \\
\text { diferencias" esto en servicio al cliente podría traducirse como persistir en los } \\
\text { productos para encontrar uno que le agrade al cliente }\end{array}$ \\
\hline $\begin{array}{l}\text { 4. Principios básicos de la } \\
\text { calidad }\end{array}$ & $\begin{array}{l}\text { 4. Ishikawa en su } 2 \text { do principio básico de la calidad menciona que "el primer } \\
\text { paso en la calidad es conocer lo que el cliente quiere" y es así que llegado el } \\
\text { momento de mostrar un producto al cliente se debe tener la certeza de que } \\
\text { sea lo que él espera }\end{array}$ \\
\hline
\end{tabular}

Fuente: Elaboración propia con base en Castillo, (2019) y Miranda, Chamorro y Rubio, (2007).

El cuarto estándar pertenece a un punto intermedio del proceso de ventas, donde el cliente ya ha indicado que desea adquirir un producto de la boutique; sin embargo, no es una garantía que la compra se concluya satisfactoriamente.

\section{Tabla 7}

Estándar 5: Adicionar

Teoría(s) para comparar

1. 14 principios de Deming

2. Trilogía de Juran
Análisis comparativo

1. Deming indica en su décimo principio que se deben eliminar las exhortaciones ya que provienen de la gerencia en forma de exigencias para los empleados, al agregar un estándar no negociable de adición de productos se compromete al asesor a ofrecer más productos de los que el cliente podría necesitar viéndose afectado en su calidad del servicio por la insistencia que el cliente pueda llegar a percibir

2. El mencionado estándar también cuenta con la característica de planificación de la calidad, pues el servicio de adición puede adecuarse a los clientes correctos, lo cual incrementa la percepción de calidad en el servicio

Fuente: Elaboración propia con base en Chacón y Rugel, (2018) y Miranda, Chamorro y Rubio, (2007).

Esta tabla se refiere a un proceso subjetivo que a percepción de un tipo de cliente puede ser positivo, pero para otros podría parecer un servicio innecesario que frustraría la compra. 
ANÁLISIS COMPARATIVO DE LOS ESTÁNDARES DE CALIDAD DE UNA EMPRESA MEXICANA CON LAS PRINCIPALES TEORÍAS DE LA CALIDAD ENFOCADAS EN EL SERVICIO AL CLIENTE.

\section{Tabla 8}

Estándar 6: Darle valor al producto

\begin{tabular}{ll}
\hline Teoría(s) para comparar & \multicolumn{1}{c}{ Análisis comparativo } \\
\hline 1. 14 principios de Deming & $\begin{array}{l}\text { 1. Al mostrar al cliente las características que hacen que el producto sea } \\
\text { especial se está cumpliendo con la filosofía de Deming de ser constante con } \\
\text { la mejora del servicio y los productos y a su vez esto se refuerza con las } \\
\text { capacitaciones y entrenamientos que la empresa ofrece a sus asesores para } \\
\text { saber de qué manera agregar valor a lo que ofrecen }\end{array}$ \\
& $\begin{array}{l}\text { 2. El asesor debe analizar y estudiar el producto que ofrece antes del proceso } \\
\text { de venta para poder recomendarlo al cliente con fundamentos sólidos y } \\
\text { concluir la venta (actuar) }\end{array}$ \\
$\begin{array}{ll}\text { 2. Ciclo PHVA (Actuar) } & \text { 3. Crosby hace referencia en su primer principio que la calidad es cumplir con } \\
& \text { los requisitos, esto se puede relacionar como mostrarle al cliente lo que } \\
\text { realmente está buscando lo cual producirá un verdadero servicio de calidad }\end{array}$ \\
$\begin{array}{ll}\text { 3. } 4 \text { principios absolutos de } \\
\text { Crosby }\end{array}$
\end{tabular}

Fuente: Elaboración propia con base en Chacón y Rugel, (2018) y Miranda, Chamorro y Rubio, (2007).

El sexto estándar establece que dar valor al producto refuerza la actitud de compra del cliente, sin embargo, es tarea del asesor cautivar al cliente con todos los rasgos positivos del producto, los cuales debe conocer desde antes de realizar el proceso de ventas.

\section{Tabla 9 \\ Estándar 7: Programa de fidelidad}

Teoría(s) para comparar

1. 14 principios de Deming

2. Ciclo PHVA (Verificar)

3. 4 principios absolutos de Crosby

4. Principios de la calidad de Ishikawa
Análisis comparativo

1. El primer principio de Edward Deming establece que se debe ser constante para mejorar los servicios y productos, esto es posible proporcionando las herramientas adecuadas y un programa de fidelidad es una buena vía de administración de los clientes y de volverlos más fieles a la marca, sin embargo, el estándar es nuevamente calificado como una exhortación u obligación para los asesores, lo que podría provocar frustración en ellos al no cumplir con el requisito de la gerencia y dificulta su proceso de servicio con el cliente.

2. Una vez que el servicio ha sido efectivo y el cliente ha percibido confianza con el asesor, la fidelización del mismo será más fácil. Esto se interpreta como la verificación del proceso de venta

3. Nuevamente se presenta la calidad en el servicio demostrando el cumplimiento de los requisitos establecidos, es decir, el asesor deberá demostrar su aptitud para fidelizar a los clientes haciendo uso del buen servicio que ofrece

4. Kaoru Ishikawa manifiesta la importancia del marketing (8vo principio) para fortalecer la relación de la empresa con los clientes, la herramienta de fidelización de los clientes con la marca tiene esa finalidad por ello la importancia de que el asesor la mencione durante el proceso de servicio

Fuente: Elaboración propia con base en Chacón y Rugel, (2018) y Miranda, Chamorro y Rubio, (2007) 
ANÁLISIS COMPARATIVO DE LOS ESTÁNDARES DE CALIDAD DE UNA EMPRESA MEXICANA CON LAS PRINCIPALES TEORÍAS DE LA CALIDAD ENFOCADAS EN EL SERVICIO AL CLIENTE.

Se muestra que el séptimo estándar es un medio ideal para fortalecer la comunicación con el cliente y dar muestra de su importancia para la empresa, lo cual puede tornarlos en clientes frecuentes, aunque también podría causar una desmotivación para el asesor que no logre fidelizar a suficientes clientes.

\section{Tabla 10}

Estándar 8: Cobro

Teoría(s) para comparar

1. Metodología 9's

2. Principios básicos de la calidad de Ishikawa

Análisis comparativo

1. El octavo estándar indica que antes de proceder al pago del producto el asesor deberá hacer una cuidadosa revisión del mismo, para asegurarse de que se encuentra en buen estado o detectar alguna anomalía; es donde se aplica la metodología 9's, el orden, la limpieza y la disciplina deberán hacerse notar tanto en el producto como en la boutique

2. Aunque el principio de la educación (1er principio) debe demostrarse desde el primer contacto con el cliente, es necesario enfatizarlo en este estándar, donde el asesor mostrará su profesionalismo, entregando un producto en excelente estado, con la envoltura adecuada y aclarando todas las dudas que el cliente pudiese tener al momento del pago y, por supuesto, reafirmando el apoyo del asesor aún después de su compra

Fuente: Elaboración propia con base en Castillo (2019) y Miranda, Chamorro y Rubio, (2007).

A pesar de no ser un estándar aplicado directamente por el asesor, es importante que este asista el proceso de cobro, comprobando que el producto se encuentra en óptimas condiciones para su venta.

\section{Tabla 11}

Estándar 9: Despedir al cliente

Teoría(s) para comparar

1. 14 principios de Deming

2. Las 7 herramientas de calidad (Diagrama de flujo)
Análisis comparativo

1. Para lograr una despedida adecuada por parte del asesor, durante la capacitación este debe sentir esa confianza y perder el miedo de tratar a los clientes como se espera por parte de la gerencia, esto lo indica Edward Deming en su octavo principio, de esta forma el cliente sentirá lo especial que es para la empresa

2. El diagrama de flujo cuenta con un inicio y un fin de procesos, despedir al cliente, se haya efectuado una compra o no, significa que concluyó el proceso de servicio que un asesor lleva a cabo

Fuente: Elaboración propia con base en Marmolejo (2019) y Miranda, Chamorro y Rubio, (2007).

En esta tabla se aprecia el final del proceso de ventas, a pesar que los 9ENN son un proceso en conjunto (diagrama de flujo), se hace énfasis en los procesos más importantes para contemplarse en las siete herramientas de calidad. 
ANÁLISIS COMPARATIVO DE LOS ESTÁNDARES DE CALIDAD DE UNA EMPRESA MEXICANA CON LAS PRINCIPALES TEORÍAS DE LA CALIDAD ENFOCADAS EN EL SERVICIO AL CLIENTE.

\section{CONCLUSIONES}

Con los resultados obtenidos mediante la comparación de los 9ENN y las teorías clásicas de los principales exponentes de la calidad, se determina que existe efectivamente una relación de conceptos básicos entre las mismas, concluyendo esto en base a la investigación y a perspectiva personal. Aún sin tener fundamentos comprobados sobre si la creación de dichos estándares está inspirada en las teorías mencionadas, se percibe una similitud de conceptos en ambas.

En la gran mayoría de los estándares se puede apreciar una relación positiva con el pensamiento de los gurús de la calidad; sin embargo, en las tablas 3, 7 y 9 se concluye que ciertas acciones en el proceso de ventas, como la adición de productos no solicitados por el cliente o requerir su información personal para uso del programa de fidelidad de la marca, podrían significar un obstáculo para el asesor, ya que son consideradas acciones forzosas $u$ obligaciones que el mismo podría percibir afectando esto en su motivación a causa del no cumplimiento de los estándares o dependiendo el tipo de cliente podría considerarse una forma hostil de acercarlo a la empresa; esto lo afirman Guadarrama y Rosales (2015) indicando que una empresa que visualiza oportunidades en todas partes no es una empresa centrada en el cliente sino en sus intereses.

Además, se observaron diferencias entre lo que establecen los padres de la calidad, se puede tomar como ejemplo el punto número 11 donde Edward Deming, (1986) opina que se deben eliminar las metas numéricas para la mano de obra... y sustituirlos por el liderazgo y la mejora continua para no perder la motivación de seguir mejorando, mientras que Philip Crosby, (1960) cree que "todos los trabajadores deben establecer objetivos individuales de mejora y metas a alcanzar en sus equipos de trabajo" (citados en Miranda, Chamorro y Rubio 2007, 34).

Se afirma entonces que las teorías de la calidad están diseñadas según las necesidades de la empresa que fundamente sus procesos en cada una de ellas, finalmente cada gurú de la calidad creó sus teorías basándose en la situación que cada uno vivía en su época, además que cabe recalcar que cada empresa actúa conforme a su mercado y su giro.

Se concluye desde el punto de vista analítico que la empresa mexicana cuenta con estándares firmes basados en la calidad, aunque, cuenta con oportunidades de mejora mínimas que sería ideal modificar para mejorar el servicio que brinda al cliente y cumplir con su misión de DÍAZ-GARCÍA A., GARCÍA-MUÑOZ APARICIO C. 
ANÁLISIS COMPARATIVO DE LOS ESTÁNDARES DE CALIDAD DE UNA EMPRESA MEXICANA CON LAS PRINCIPALES TEORÍAS DE LA CALIDAD ENFOCADAS EN EL SERVICIO AL CLIENTE.

"encontrar la manera de establecer relaciones reales y de largo plazo" (Ramírez, 2020) como lo indicó su Director Comercial.

Por último, en vista de las limitaciones al encontrar la información necesaria, sería recomendable realizar más investigaciones de la calidad enfocada al servicio al cliente, pues en la actualidad una de las características con mayor exigencia por los clientes es el buen servicio, la calidez, la confianza y la experiencia que una empresa pueda brindarle. Tal como indican Solórzano y Aceves (2013):

La calidad en el servicio al cliente es uno de los puntos clave para permanecer entre la preferencia de los consumidores, dicho que esta es la imagen que se proyecta en ellos; siendo a su vez un diferenciador ante la competencia, y es el punto decisivo en el cual dichos clientes se crean una opinión positiva o negativa sobre la organización.

Además, se debe reconocer la necesidad de la mayoría de las empresas estatales y nacionales de capacitar a todos sus niveles para transmitir a los individuos esa sensación de sentirse parte de la empresa y llevarse a casa una experiencia agradable.

\section{REFERENCIAS BIBLIOGRÁFICAS}

Alvarado, B. y Rivas, G. (2006). Philip Crosby. Estrategias de calidad y competitividad. Recuperado de https://www.geocities.ws/chex88chex/estrategia/PhilipCrosby.pdf

Anderson, J., Rungtusanatham, M. y Schroeder, R. (1994). A theory of quality management underlying the Deming Management Method, Academy of Management Review, 19(3), 472-509.

Barajas, C (2011). Estándares de venta. [Manual de políticas y procedimientos]. México: Prada. Barajas, C (2013). Manual de asesor de ventas. [Material de capacitación]. México: Prada. 
ANÁLISIS COMPARATIVO DE LOS ESTÁNDARES DE CALIDAD DE UNA EMPRESA MEXICANA CON LAS PRINCIPALES TEORÍAS DE LA CALIDAD ENFOCADAS EN EL SERVICIO AL CLIENTE.

Castillo, S. (2016). Metodología de las 9s en el área de patrocinio de la organización comunitaria Nuevo Amanecer. (Tesis de maestría). Pontificia Universidad Católica del Ecuador, Ecuador. Recuperado de https://repositorio.pucese.edu.ec/bitstream/123456789/636/1/CASTILLO\%20IZQUIERDO \%20SEGUNDO\%20ERIBERTO.pdf

Chacón, J. y Rugel, S. (2018). Artículo de revisión. Teorías, modelos y sistemas de gestión de calidad. Revista Espacios. 39(50). 14-22. Recuperado de http://www.revistaespacios.com/a18v39n50/a18v39n50p14.pdf

Crosby, P. (1988). La organización permanece exitosa. México: McGraw-Hill.

Deming, E. (1989). Calidad, productividad y competitividad a la salida de la crisis. Madrid: Editorial Díaz de Santos.

Esencia Prada. (s.f). Esencia Prada. [Página web]. Prada. Recuperado de https://prada.mx/esencia-prada [Consultado el 22 de junio de 2020]

Guadarrama, E. y Rosales, E. (2015). Marketing relacional: Valor, satisfacción, lealtad y retención del cliente. Análisis y reflexión teórica. Ciencia y Sociedad. 40(2). 307-340. Recuperado de https://www.redalyc.org/pdf/870/87041161004.pdf

Hatch, H. (2019). El $82 \%$ de los clientes se pierden por mala atención. Soy.marketing. Recuperado de: https://soy.marketing/el-82-de-los-clientes-se-pierden-por-mala-atencion/

Hernández, R., Fernández, C. y Baptista, P. (2014). Metodología de la investigación. (6ª Ed.). México: McGraw-Hill Interamericana. 80.

Ishikawa, K. (1986). ¿Qué es control total de la calidad? Colombia: Editorial normal.

Juran, J. (1990). Juran y la planificación de la calidad. Madrid: Editorial Díaz de Santos.

Marmolejo, J. (2019). Las 7 herramientas básicas de la calidad. SCP Consulting group. Recuperado de https://spcgroup.com.mx/las-7-herramientas-basicas-de-la-calidad/

Martínez, M. (2019) La investigación cualitativa (síntesis conceptual). Revista IIPSI. 9(1). 123146.

Recuperado

de https://pdfs.semanticscholar.org/6691/f8bdfca2be9adc1b98d757e02c3af08d27ba.pdf

Miranda, F., Chamorro, A. y Rubio, S. (2007). Introducción a la gestión de la calidad. Delta publicaciones: Madrid. Recuperado de 
ANÁLISIS COMPARATIVO DE LOS ESTÁNDARES DE CALIDAD DE UNA EMPRESA MEXICANA CON LAS PRINCIPALES TEORIIAS DE LA CALIDAD ENFOCADAS EN EL SERVICIO AL CLIENTE.

https://books.google.com.mx/books?id=KYSMQQyQAbYC\&printsec=frontcover\&dq=introd uccion+a+la+gestion+de+la+calidad\&hl=es-

419\&sa=X\&ved=2ahUKEwjPtpaZ4oPrAhUPjq0KHUfVDKwQ6AEwAHoECAAQAg\#v=onep age\&q=introduccion\%20a\%20la\%20gestion\%20de\%20la\%20calidad\&f=false

Ojeda, M. y López, L. (2000). Deming: la revolución de la calidad y las herramientas de la estadística. La ciencia y el hombre. 8(1) 1-11. Recuperado de https://www.uv.mx/ personal/mojeda/files/2012/04/Deming_LaRevoluciondelacalidad.pdf

Ramírez, S. (2020). La firma mexicana Prada lleva la boutique a tu casa. [Revista web] Forbes México. Recuperado de https://www.forbes.com.mx/forbes-life/la-firma-mexicana-pradalleva-la-boutique-a-tu-casa/amp/

Ruiza, M., Fernández, T. y Tamaro, E. (2004). Biografía de Kaoru Ishikawa. Biografías y Vidas. La enciclopedia biográfica en línea. Barcelona: España. Recuperado de https://www.biografiasyvidas.com/biografia/i/ishikawa.htm

Schnarch, A. (2011). Marketing de fidelización: Como obtener clientes satisfechos y leales, bajo una perspectiva latinoamericana. Ecoe Ediciones: Colombia. 13.

Solórzano, G. y Aceves, J. (2013). Importancia de la calidad del servicio al cliente para el funcionamiento de las empresas. El Buzón de Pacioli. 8(82) 4-13. Recuperado de https://www.itson.mx/publicaciones/pacioli/Documents/no82/pacioli-82.pdf

Tovar, P. (2017). Shigeo Shingo: Biografía, Aportes y Filosofía de Calidad. Lifeder.com. Recuperado de https://www.lifeder.com/shigeo-shingo/ 